\title{
Data-driven research on chemical features of Jingdezhen and Longquan celadon by energy dispersive X-ray fluorescence
}

\author{
Ziyang $\mathrm{He}^{\mathrm{a}, \grave{\dagger}, *,}$, Maolin Zhang ${ }^{\mathrm{b}, \dagger}$, Haozhe Zhang ${ }^{\mathrm{c}}$ \\ ${ }^{a}$ Department of Industrial and Manufacturing System Engineering, \\ Iowa State University, Ames, IA 50011 USA. \\ b Jingdezhen Ceramic Institute, Jingdezhen 333001, China. \\ ${ }^{\mathrm{c}}$ Department of Statistics, Iowa State University, Ames, IA 50011 USA
}

${ }^{\dagger}$ Z.H. and M.Z. are joint first authors.

${ }^{*}$ Corresponding Author:

Ziyang He

Email: heziyang@iastate.edu

Address:

Department of Industrial and Manufacturing System Engineering, Iowa State University, Ames, Iowa 50014, USA. 
Abstract The energy dispersive X-ray fluorescence (EDXRF) is used to determine the chemical composition of celadon body and glaze in Longquan kiln (at Dayao County) and Jingdezhen kiln. Forty typical shards in four cultural eras were selected to investigate the raw materials and firing technology. Random forests, a relatively new statistical technique, has been adopted to identify chemical elements that are strongest explanatory variables to classify samples into different cultural eras and kilns. The results indicated that the contents of $\mathrm{Na}_{2} \mathrm{O}, \mathrm{Fe}_{2} \mathrm{O}_{3}, \mathrm{TiO}_{2}$, $\mathrm{SiO}_{2}$ and $\mathrm{CaO}$ vary in celadon bodies from Longquan and Jingdezhen, which implies that local clay was used to manufacture celadon bodies in Jingdezhen kiln. By comparing the chemical composition in glaze, we find that the chemical elements and firing technology of Jingdezhen kiln are very similar to those in Longquan kiln, especially for Ming dynasty. This study reveals the inheritance between Jingdezhen kiln and Longquan kiln, and explains the differences between those two kilns.

Key words: celadon, EDXRF, chemical feature, Longquan, Jingdezhen, random forests.

\section{Introduction}

Celadon is one of the earliest porcelains in China as well as in the world. With its long history and rich varieties, celadon has attracted a lot of attentions from researchers in archaeology, physics, material science, etc. Longquan kiln, one of the most representative kilns in celadon production, enjoys an important status in Chinese ceramic history [1]. The government in Ming Dynasty invested nationwide labors, resources and capitals in the establishment of imperial kiln in Jingdezhen from Yongle era. Jingdezhen has also became the craftsmen aggregation center and the porcelain manufacturing center in Maritime Silk Road since Yongle era of Ming Dynasty [2]. Jingdezhen kiln were able to make color glaze and whiteware porcelains from Yuan Dynasty and began to make celadon from Tang Dynasty. The demand from commercial activities, foreign trade and imperial reward far stripped the supply of Jingdezhen porcelain [3]. Due to the good quality and unique glaze feature (jade texture) of Longquan celadon, Jingdezhen kiln was trying to imitate Longquan celadon from style, color and pattern. The producing and firing technology of imitated Longquan celadon in Jingdezhen had reached a relative high level in Yongle era of Ming Dynasty (1403 - 1424), however craftsmen did not master the completely mature firing technology until Yongzheng era of Qing Dynasty (1723 - 1735) [4]. At present, Longquan celadon has been in depth studied by current archaeologist while the imitated Longquan celadon in Jingdezhen still 
remains little investigated.

It has been well known that the contents of macro and micro elements in the body and glaze of porcelain are dependent on the raw material and firing technology [5, 6]. Li et al. [7] used EDXRF to confirm that the raw materials of imperial Longquan porcelain have no obvious changes between Hongwu and Yongle era (1368 - 1398), and claimed the firing technology has not declined in the Early Ming Dynasty. Zhu et al. (2011) [8] shows micro elements, e.g. Cr, Sr, Zr in body and Sr, $\mathrm{Rb}$ in glaze could be employed as relevant markers for the non-destructive discrimination of the provenance of Xicun and Yaozhou kiln. Wu et al. (2015) [9] is a research on the early celadon of Jingdezhen and its initial development. It comparatively analyzed the EDXRF data from Nan and Lantin kiln sites in Jingdezhen and explained the difference in chemical composition and processing characteristics.

In this study, the samples of body and glaze of Longquan celadon in Northern Song, Southern Song, Yuan and Ming Dynasties as well as imitated Longquan celadon in Jingdezhen in Ming Dynasty were examined by the energy dispersive X-ray fluorescence (EDXRF) microprobe. EDXRF is an efficient instrument that combines the merits of X-ray fluorescence spectrometry with the ability to analyze a micro-area. The large chamber size allows for the non-destructive analysis of macro and trace elements on micro-areas of the samples. After obtaining the data, Random forests, a well-established machine learning algorithm, was adopted in the analysis, rather than multivariate statistical methods. If many elements were measured in the experiments, it's very inefficient to find the difference by standard multivariate statistical methods (Li et al. 2010 [10]). However, Random forests can return the values that measure the variable importance of explanatory variables in the training data, so only the top-ranked elements need to be investigated. The results of the analysis reveal the inheritance relationship of the celadon from Longquan and Jingdezhen kiln.

\section{Experiment}

In this experiment, 40 samples of Longquan celadon in Northern Song, Southern Song, Yuan and Ming Dynasties as well as imitated Longquan celadon in Jingdezhen civilian kilns in Ming Dynasty are collected. They include 7 samples of Longquan celadon in Northern Song Dynasty (LQ-BS1 LQ-BS-7), 8 samples of Longquan celadon in Southern Song Dynasty(LQ-NS-1 LQ-NS-8), 9 samples of Longquan celadon in Yuan Dynasty(LQ-Y-1 LQ-Y-9), 3 samples of Longquan celadon in Ming Dynasty(LQ-M-1 LQ-M-3) and 13 samples of imitated Longquan celadon in Jingdezhen 
in Ming Dynasty(FLQ-M-1 FLQ-M-13). The photographs of samples are shown in Figure 2.

The samples were cut and cleaned in an ultrasonic bath and then dried before testing. Energy Dispersive X-ray Fluorescence (EDXRF) spectrometer (EAGLE-III) was used to detected body and glaze compositions. The analysis was executed at $50 \mathrm{kV}$ and $200 \mathrm{~mA}$ voltage-current of the X-ray tube, with a vacuum optical route and dead time was around $25 \%$. The detector is a liquidnitrogen-cooled $\mathrm{Si}(\mathrm{Li})$ crystal with $\mathrm{Rh}$ window, and the beam spot was $300 \mu \mathrm{m}$. The software employed for spectrum retraction and analysis was the program VISION32, associating with the instrument. Quantitative analysis was operated by calibration curve method. The calibration samples were from a set of reference samples (13 pieces) with known chemical compositions, provided by the Shanghai Institute of Ceramics of the Chinese Academy of Science (SICCAS). The analytical results were in Table 1 and 2 .

The firing temperatures of the typical samples were estimated from the inflection point of the thermal expansion curves (Table 3) and measured by DIL 402C Thermal Dilatometer of the German NETZSCH Instrument Company. Water absorptions were tested through boiling method.

\section{Results and discussion}

\subsection{Random forests and variable importance}

Random forests, an increasingly popular nonparametric methodology, is an extension of classification and regression trees (CART) method [11]. It grows many classification trees or regression trees and thus has the name "forests". Every tree is built using a deterministic algorithm, and the trees are different in two aspects: First, at each node, a best split is chosen from a random subset of the predictors rather than all of them; Second, every tree is built using a bootstrap sample of the observations. The remaining sample, the so-called "out-of-bag" (OOB) sample, which contains approximately one-third of the observations, are then used to estimate the prediction accuracy. A key feature of random forest is its ability to measure variable importance [12]. Variable importance reflects the degree of association between a given explanatory variable and the response variable. More details about random forests and its wide applications can be found in $[13,14]$.

In this research, the contents of chemical elements are the explanatory variables and the categories of eras and kilns are the response variable. By using randomForest Package in $\mathrm{R}$ language [15], we performed random forests method to do classification of celadon samples. Mean descrease in accuracy (MDA) and mean decrease in Gini index (MDG), two values that random forests method returns after constructing classification trees, can be used to identify chemical 
elements that differ most significantly among goups of samples in different categories of ears and kilns. The returned values of MDA and MDG are shown in Table 4 and 5. Random forests method is more efficient in selecting important variables than standard multivariate statistical methods, such as principal component analysis and linear regression, especially when the number of measured chemical elements is large [10].

\subsection{Analysis of the chemical composition of the celadon body in Longquan and Jingdezhen}

The data in Table 1 show obvious differences in compositions of bodies between Longquan and Jingdezhen kilns. It can be seen that the $\mathrm{Al}_{2} \mathrm{O}_{3}$ contents of celadon body in Jingdezhen (19.62\%) are lower than those in Longquan (22.90\%), whereas the $\mathrm{SiO}_{2}$ contents of celadon body in Jingdezhen $(72.40 \%)$ are higher than those of the Longquan (67.93\%). The average contents of $\left(\mathrm{RO}+\mathrm{R}_{2} \mathrm{O}\right)$ are $5.70 \%$ in Jingdezhen and $6.27 \%$ in Longquan. The flux contents in two kilns don't display any significant differences. The only difference is that Jingdezhen kiln is in high silicon flux domain, but Longquan kiln is in low silicon flux domain. Meanwhile, together with results from sintering temperature test (see Table 3), it can been shown that the average sintering temperature of celadon is $(1139.75 \pm 20){ }^{\circ} \mathrm{C}$ in Jingdezhen kiln and mainly between $1157{ }^{\circ} \mathrm{C}$ and $1230{ }^{\circ} \mathrm{C}$ in Longquan kiln. In order to meet the demand of fully sintered body, average sintering temperature of celadon in Jingdezhen is slightly lower than that in Longquan.

In our research, EDXRF data of the bodies in different cultural eras are investigated. We did leave-one-out cross-validation [16] of chemical composition data by using Random forests. The first main conclusion is that there is no obvious difference among different cultural eras in Longquan kiln, since the mis-classification ratio of cross-validation is $52.2 \%$. Another important conclusion is that low mis-classification ratio (2.6\%) shows clear difference between samples from two kilns. Table 4 shows variable importance of observed data. In macro-element domain, $\mathrm{Fe}_{2} \mathrm{O}_{3}$ $(\mathrm{MDG}=3.7851)$ and $\mathrm{CaO}(\mathrm{MDG}=2.7215)$ are two most important elements to classify origins of products in Longquan and Jingdezhen. In the micro-element domain, $\mathrm{ZrO}_{2}(\mathrm{MDG}=4.0653)$ and $\mathrm{Y}_{2} \mathrm{O}_{3}(\mathrm{MDG}=2.3640)$ are other two important elements.

As displayed in Figure 3, the celadon bodies from Jingdezhen contain more CaO than Longquan celadon and have a relatively large fluctuating range. The bodies from Longquan own higher contents of $\mathrm{Fe}_{2} \mathrm{O}_{3}$ than those bodies from Jingdezhen and also have a wide fluctuating range. Bodies sampled from Jingdezhen were dispersed in lower left domain, while bodies sampled from 
Longquan were in lower right domain. The contents of $\mathrm{Fe}_{2} \mathrm{O}_{3}$ in Longquan samples in different cultural eras can show the continuous inheritance relationship of body formula evolution. It has been confirmed by the data from archeological research that Southern Song Dynasty kiln site is in the vicinity of Ming Dynasty kiln site [17]. This has also indicated that the albite contents are high in raw materials of Jingdezhen kiln whereas potassium feldspars are the main raw materials of Longquan kiln, which can directly be seen from Table 6 [18] [19].

The iron and titanium contents are usually higher in samples from Longquan kiln than those from Jingdezhen kiln, as can be seen in Table 6, raw materials in Jingdezhen are featured by lower iron and titanium contents. The average $\mathrm{Fe}_{2} \mathrm{O}_{3}$ content in Mingsha kaolin soil and Xingzi kaolin soil is $1.335 \%$ and the average $\mathrm{TiO}_{2}$ content is $0.025 \%$, which are much lower than those of Longquan kiln soil (Taking Zijin soil in the Dayao County Gaojitou village as an example). The average $\mathrm{Fe}_{2} \mathrm{O}_{3}$ content is $3.11 \%$ and average $\mathrm{TiO}_{2}$ content is $0.45 \%$, respectively. From the appearance of the samples, most celadon bodies from Longquan are gray while bodies from Jingdezhen are mainly white.

In conclusion, the main reasons for the differences mentioned above can be listed as followed. Firstly, the body element is influenced by local raw materials and addition of Zijin soil. Compared with Jingdezhen kiln, Longquan kiln displays the features of high aluminum and iron with low silicon. This is mainly due to the fact that Zijin soil has high contents of iron and aluminum was added during bodies manufacturing. Secondly, the selection of raw materials for making celadon bodies is limited by local resources and has to tailor to local conditions. From excavated archeological data [17], the raw materials for making porcelain was obtained in the vicinity of the two kiln sites, thus raw materials in distinct kilns remained different. With consideration for cost, the craftsmen in Jingdezhen were unable to acquire the celadon body raw materials in great demand from distant areas. Consequently, at that time they employed the local raw materials to imitate Longquan celadon, which resulted in the differences in chemical compositions in two kilns' production.

\subsection{Analysis of the chemical composition of the celadon glaze in Longquan and Jingdezhen}

Longquan celadon glaze can be roughly divided into two categories according to the features. One category is the transparent glaze with high gloss, which could be represented by the glaze in Northern Song Dynasty; the other category is the jade texture glaze, which could be represented 
by the glaze in Southern Song Dynasty [20]. Glaze from Jingdezhen kiln shares similar features. From Table 2, the CaO contents of Longquan celadon in Northern Song Dynasty (10.75\%) were significantly higher than those of the Jingdezhen in Ming Dynasty (6.20\%) as well as those of Longquan kiln in other cultural eras $(7.47 \%)$, while $\mathrm{R}_{2} \mathrm{O}$ contents are significantly lower and only reach $4.48 \%$. As Seen from the samples appearances, the glaze of Longquan celadon in Northern Song Dynasty is glossier and more transparent. Carved patterns can be clearly seen and these samples do have smoke absorption phenomenon.

As aforementioned, random forests has also been used to analyze EDXRF data of glaze samples. There is still no obvious difference between different cultural eras in glaze samples from Longquan kiln, since the mis-classification ratio of cross-validation is $53.2 \%$. Table 5 shows variable importance of chemical elements in glaze. In marco-element region, $\mathrm{Na}_{2} \mathrm{O}$ (2.1794) and $\mathrm{CaO}$ (1.9602) are the two most important macro-elements; $\mathrm{SrO}$ (4.3968) and $\mathrm{ZrO}_{2}$ (3.3247) are two other important micro-elements variables. Consitent with that Li et al. (2010) [10] claiming $\mathrm{Sr}$ isotopic and trace chemical features of whiteware shards can be linear array rule to distinguish Nanwan and three other sites in the Erlitou culture period.

From the scatterplot of $\mathrm{SrO}$ and $\mathrm{ZrO}_{2}$ (Figure 5), it can be seen that the points of samples from Longquan kiln are in the right upper part and the points of samples from Jingdezhen kiln are in left lower part. Compared with Figure 4, the macro-element $\mathrm{CaO}$ and $\mathrm{Na}_{2} \mathrm{O}$ domains of Longquan kiln have some overlapping parts with Jingdezhen kiln, especially in Southern Song and Yuan Dynasty. This indicated the formulas the craftsmen in Jingdezhen were using to imitate, and micro-element level could tell the difference of these formulas, which is reasonable to believe due to different origins and technical modification.

From Southern Song Dynasty to Ming Dynasty, $\mathrm{R}_{2} \mathrm{O}$ contents of Longquan celadon show obvious increase while $\mathrm{CaO}$ contents display gradual reduction (Table 2). $\mathrm{R}_{2} \mathrm{O}$ contents of Longquan celadon reached a peak (5.98\%) in Ming Dynasty with the $\mathrm{CaO}$ contents of $6.20 \%$. It indicated that the chemical composition of Longquan celadon glaze had successfully transformed from calcium glaze to calcium-alkali glaze [21].

Furthernore, compared with the high similarity (cultural eras continuity) among the glaze samples from Longquan kiln, the imitated celadon glaze from Jingdezhen can be divided into three categories [17]. The proper growth in $\mathrm{N}_{2} \mathrm{O}$ contents can lower the melting temperature of glaze, broaden the melting temperature variation and increase the high temperature viscosity to retain more bubbles and un-melted quartz which could improve the jade texture of celadon [6]. At the same time, the firing temperature of celadon with better jade texture is usually at the lower 
bound of positive combustion. Once the firing temperature enters the over firing temperature variation, the glaze will become transparent and glossy and lose its jade texture [20]. As can be seen in Table 6, the firing temperature of Jingdezhen celadon is around $1140^{\circ} \mathrm{C}$, this is much lower than that of ordinary ceramics $\left(1250^{\circ} \mathrm{C}\right)$ in Jingdezhen and a bit lower than that of Longquan celadon $\left(1174^{\circ} \mathrm{C}\right)$. The assumption is the craftsmen made some adjustments on purpose to develop a better jade texture for the glaze due to the uniqueness of celadon. The average contents of alkali metal oxides in imitated celadon in Jingdezhen are clearly higher than those of Longquan celadon. Some samples (FLQ-12) with high $\mathrm{R}_{2} \mathrm{O}$ have irregular cracks in the glaze layers, mainly due to the relatively high $\mathrm{Na}_{2} \mathrm{O}$ contents in the glaze [22].

As shown in Figure 6, the contents of $\mathrm{Fe}_{2} \mathrm{O}_{3}$ and $\mathrm{TiO}_{2}$ in celadon samples from Jingdezhen are very similar but much higher than those of Longquan celadon in Northern Song and Southern Song Dynasty. This reveals that the craftsmen at that time knew the formula of Longquan celadon well and also found alike raw materials [23], for instance, the Zijin soil with high contents of iron. In the meantime, the $\mathrm{Na}_{2} \mathrm{O}$ contents (1.01\%) in imitated Longquan celadon in Jingdezhen in Ming Dynasty are different from those in Longquan celadon in Ming Dynasty (Figure 6), which further explains that the Jingdezhen adopted a kind of local raw materials that are not completely same with that in Longquan.

\section{Conclusion}

By analyzing data of body and glaze chemical compositions, we obtained the following conclusions. There are some differences in the body chemical compositions between the imitated Longquan celadon in Jingdezhen and Longquan celadon. The body of imitated Longquan celadon in Jingdezhen has comparatively high contents of silicon and low contents of iron and titanium, which is similar to the feature of raw material in Jingdezhen location. It shows that Jingdezhen craftsmen acquired the celadon bodies' raw materials in great demand from the local area during the imitated Longquan celadon producing.

Imitated Longquan celadon in Jingdezhen directly or indirectly learned producing technology from Longquan celadon. Jingdezhen celadon is similar to Longquan celadon both in glaze chemical composition and firing technology. Jingdezhen craftsmen not only sought for the similar glaze raw materials in local area but also employed the firing temperature of around $1140^{\circ} \mathrm{C}$ to produce the imitated glazes. This firing temperature is close to that of Longquan celadon $\left(1174^{\circ} \mathrm{C}\right)$ but obviously much lower than that of ordinary ceramics $\left(1250^{\circ} \mathrm{C}\right)$ in Jingdezhen. 
The chemical compositions of imitated Longquan celadon glaze in Jingdezhen can be divided into three categories. One has much higher contents of alkali metal oxide in some samples than Longquan celadon glaze. They have more cracks in the glaze layers. The other two categories are respectively consistent with the features of Longquan celadon glaze in Northern Song, Southern Song and Ming Dynasties.

\section{Acknowledgements}

M. Zhang's research was partially supported by the National Science Foundation of China (51362017 and 11205073) and Social Science Funds of Jiangxi Province (2010GZC0088).

\section{References}

[1] K. P. Xiang. Longquan Celadon Research Review. Relics from Oriental Cultural. 26 (2008) 38.

[2] B. Q. Zhu. The Light industry Hall of Zhejiang Province. Study on Longquan celadon. Cultural Relics Press. Beijing. 1989. 1-37.

[3] H. L. Yang. Review of Jingdezhen intimated Longquan Celadon. Relics from Jiangxi. 4 (1991) 78.

[4] The Chinese Ceramic Society. History of Chinese Pottery and Porcelain. Cultural Relics Press. Beijing. 1982. 386.

[5] J. M. Wu, M. L. Zhang, Q. J. Li. Chemical Composition and Chromaticity Characteristic of Jilan Glaze of Ming and Qing Official Kilns. Spectroscopy and Spectral Analysis. 32 (8) (2012) 2254.

[6] F. K. Zhang. Scientific and Technical Research Achievements in Ancient Chinese Pottery and Porcelain. Shanghai Science and Technology Press. Shanghai. 1985. 161.

[7] L. Li, S. L. Feng, X. Q. Feng, Q. Xu, L. T. Yan, B. Ma, L. Liu. Study on chemical features of Longquan celadon at Fengdongyan kiln site in Yuan and Ming Dynasties by EDXRF, Nuclear Instruments and Methods in Physics Research B. 292 (2012) 25-29. 
[8] T. Q. Zhu, H. Huang, H. M. Wang, L. M. Hu, X. B. Yi. Comparison of celadon from the Yaozhou and Xicun kilns in the Northern Song Dynasty of China by X-ray fluorescence and microscopy. Journal of Archaeological Science. 38 (2011) 3134-3140.

[9] J. Wu, M. L. Zhang, T. J. Hou, Q. J. Li, J. M. Wu. Analysis of the celadon of the Tang and the Five Dynasties unearthed from Nan kiln and Lantian kiln site of Jingdezhen. Ceramics International. 41 (2015) 6851-6857.

[10] B. P. Li, L. Liu, X. C. Chen, J. X. Zhao, J. Drennan, A. Greig, Y. X. Feng, M. Lawrence, H. Xu, G. H. Han, J. X. Zhu, S. L. Zhang. Chemical comparison of rare Chinese white pottery from four sites of the Erlitou state: results and archaeometrical implications. 5 (2010) 760-776.

[11] L. Breiman, Random Forests. Machine Learning. 1. 25 (2001) 5-32.

[12] L. Breiman, J. Friedman, C. J. Stone, R. A. Olshen. Classification and regression trees. CRC press, 1984.

[13] R. Xu, D. Nettleton, D. J. Nordman. Case-Specific Random Forests. Journal of Computational and Graphical Statistics. (2015) In press.

[14] J. J. O’ Brien, P. G. Spry, D. Nettleton, R. Xu, G. S. Teale. Using Random Forests to distinguish gahnite compositions as an exploration guide to Broken Hill-type PbZnAg deposits in the Broken Hill domain, Australia. Journal of Geochemical Exploration, 149, 2015: 74-86.

[15] A. Liaw, M. Wiener. Classification and regression by randomForest. R news, 2(3), 2002:18-22.

[16] G. H. Golub, M. Heath, G. Wahba. Generalized cross-validation as a method for choosing a good ridge parameter. Technometrics, 21(2), 1979: 215-223.

[17] B. Peng, S. Zhou, Y. Shen, B. Li. EDXRF study on Ming Dynasty celadons' sherds unearthed from Longquan Feng Dong Rock, Journal of the Chinese Ceramic Society. 11. 37 (2009) 19041908.

[18] R. Zhou, Y. Y. Guo, M. Y. Wan. Research on the Raw Materials of Longquan celadon. Cultural Relics Press. Beijing. 1998. 133.

[19] Y. Y. Guo. Scientific and Technical Research Achievements in Ancient Chinese Pottery and Porcelain. Shanghai Science and Technology Press. Shanghai. 1985. 286. 
[20] J. Z. Li. Chinese History of Science and Technology. Volume of Ceramics, Science Press. Beijing. 1998. 301.

[21] H.J. Luo. The criterion for compartmentalizing the Chinese ancient glaze and its application in research of ancient glaze. Journal of the Chinese Ceramic Society. 2 (1995) 50-54.

[22] J. J. Li. Ceramics Technology, China Light Industry Press. Beijing. 2009. 87. 168

[23] L. Yuan, X. Gao, J. Dong, B. Kang, J. Miao. A Preliminary study of the Ming and Qing Guan-typed wares collected in the Palace Museum. Palace Museum Journal. 5 (2010) 184. 


\section{Figures and tables}

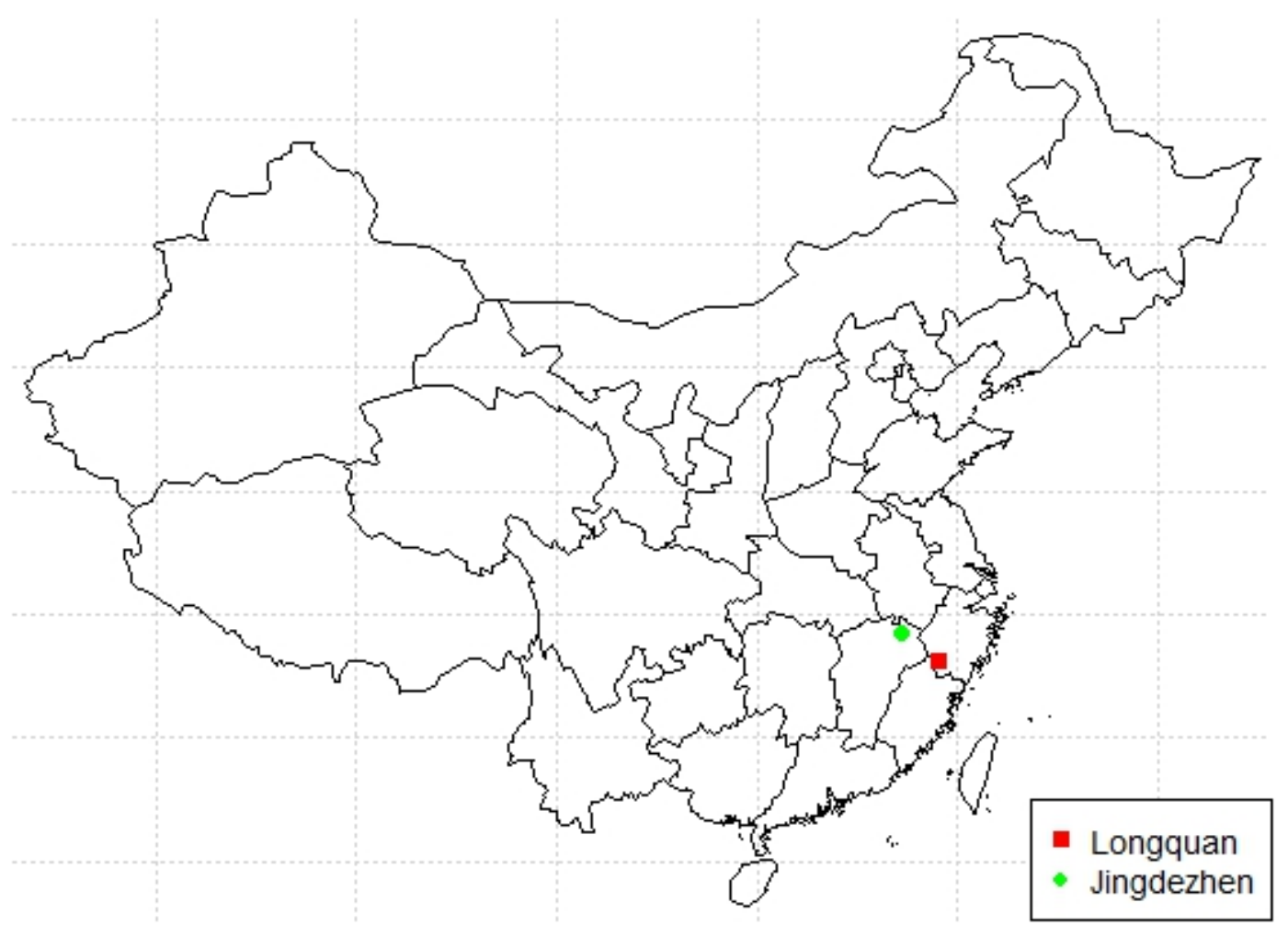

Fig. 1: The locations of Longquan kiln (Dayao County) and Jingdezhen kiln 

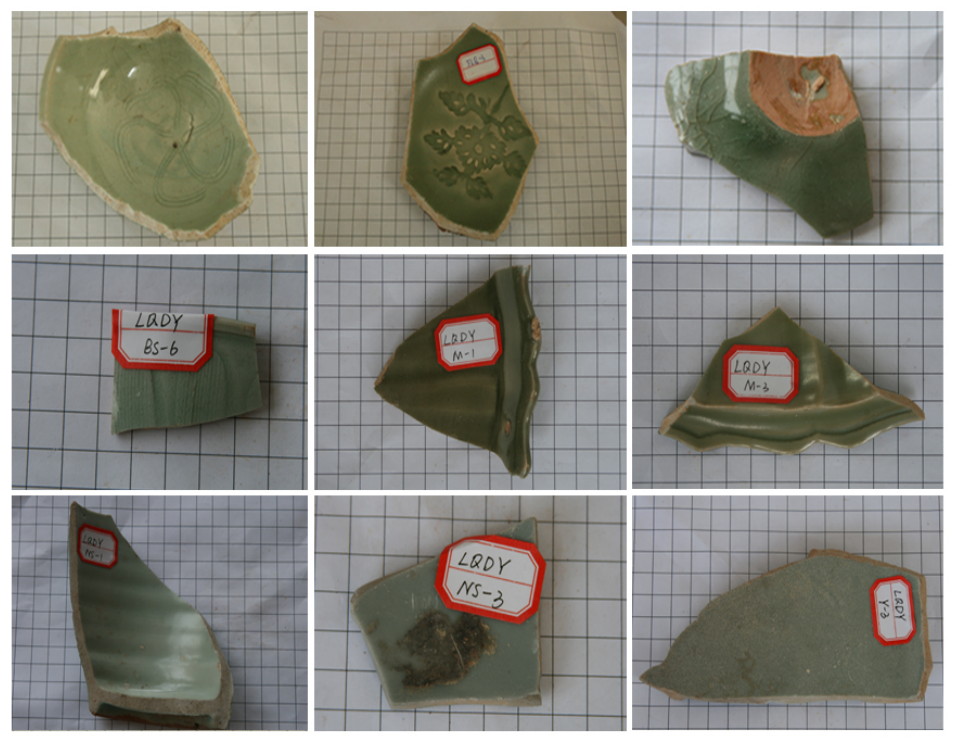

Fig. 2: Photographs of celadon samples from the Longquan kiln and Jingdezhen kiln

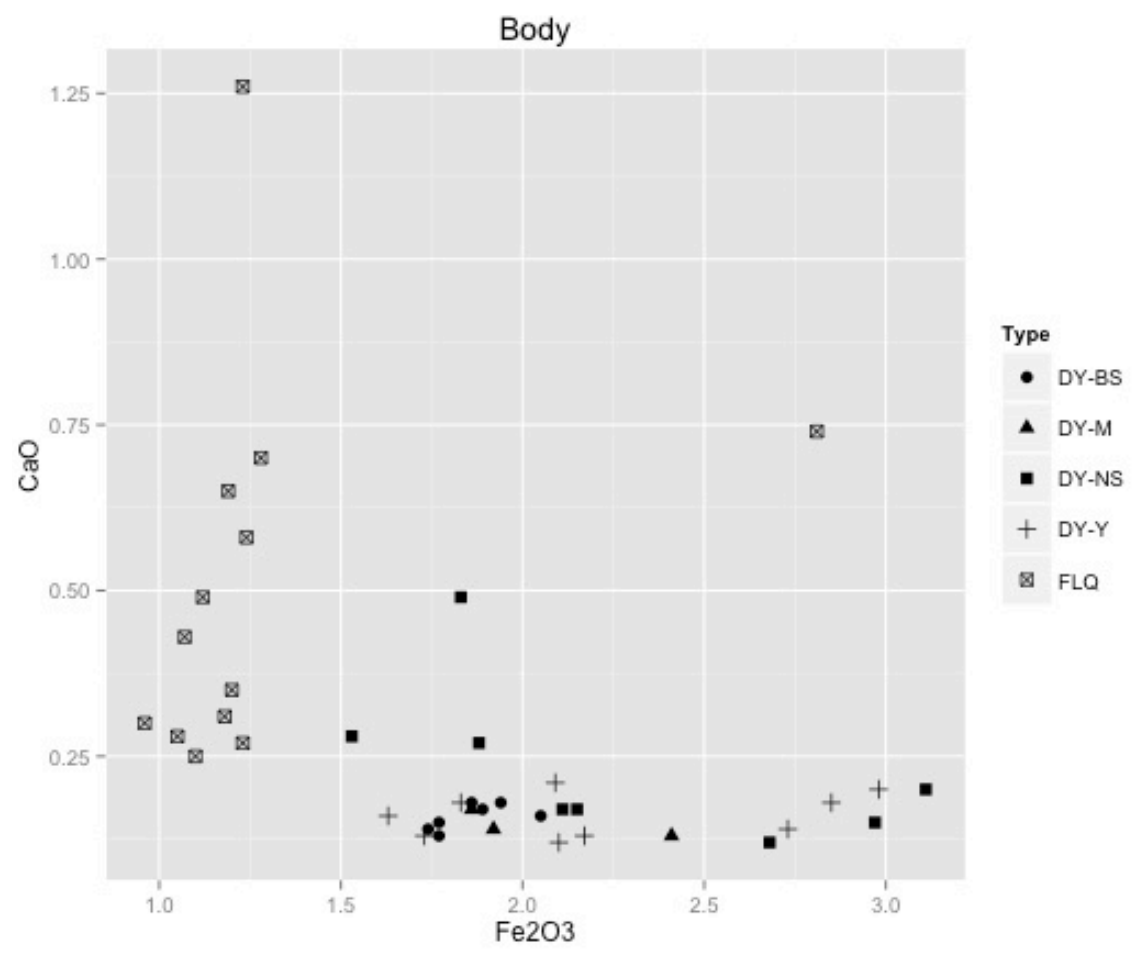

Fig. 3: The scatterplot of macro-elements in the bodies in different cultural eras and kilns. 


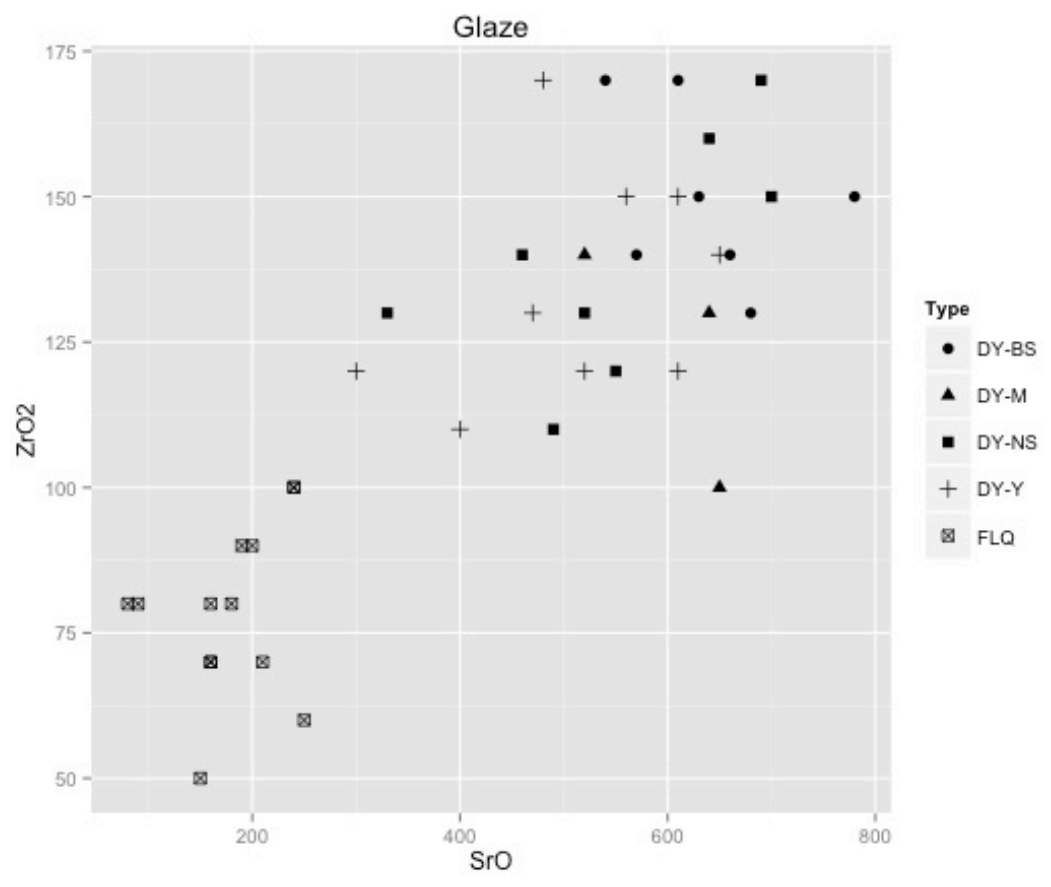

Fig. 4: The scatterplot of micro-elements of the glaze in different cultural eras and kilns

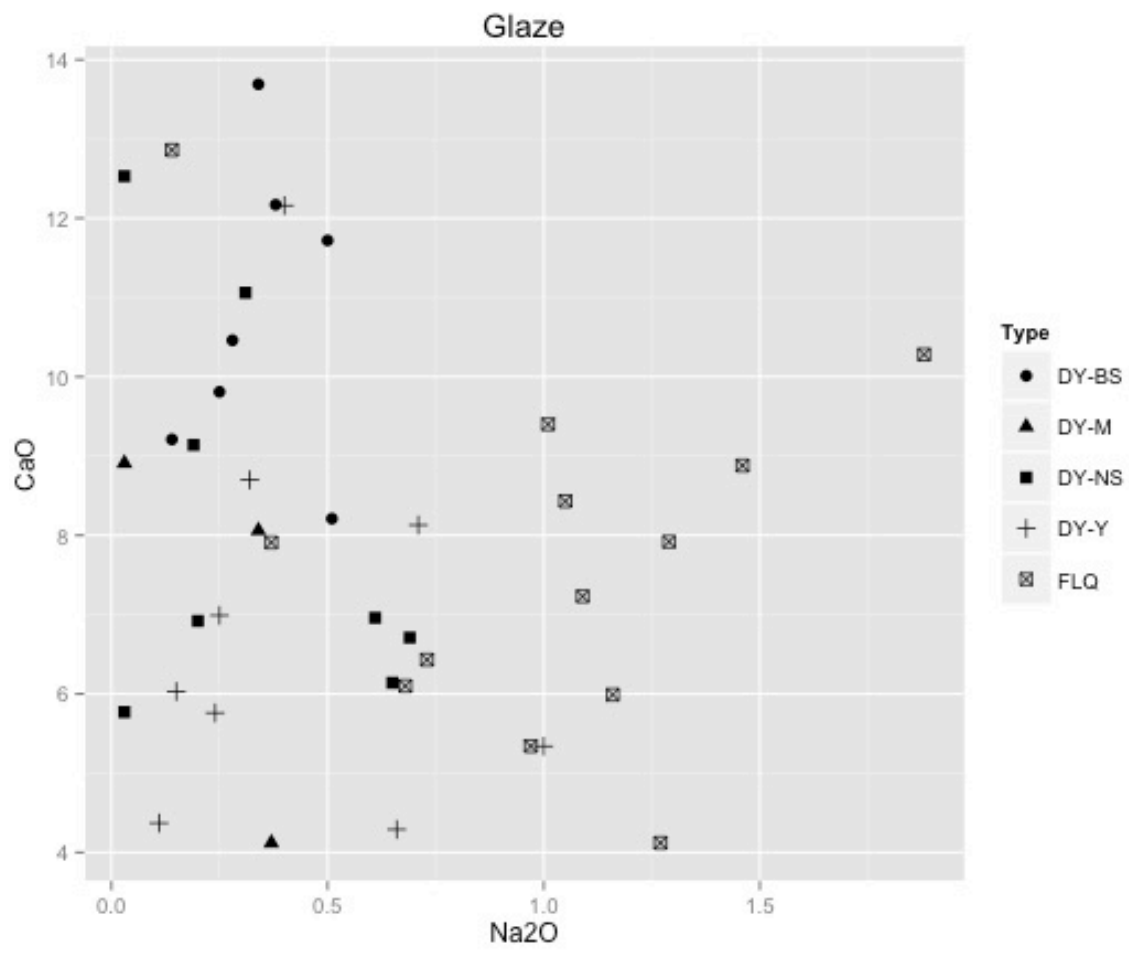

Fig. 5: The scatterplot of macro-elements of the glaze in different cultural eras and kilns 


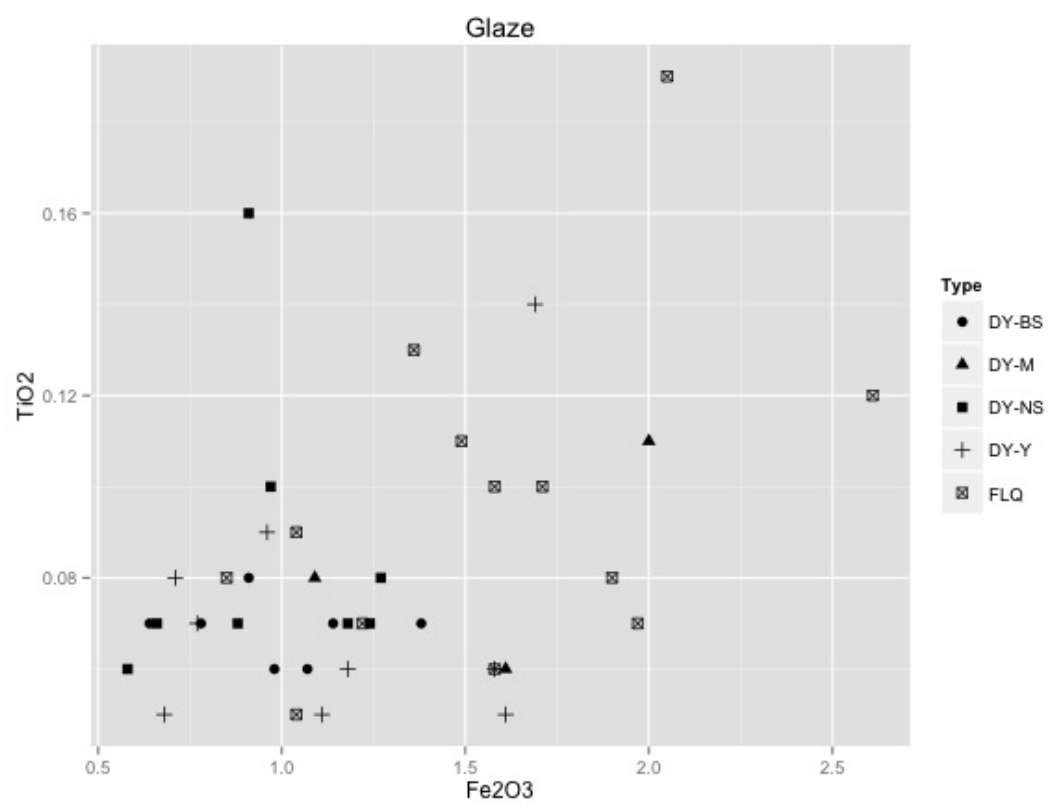

Fig. 6: The scatterplot of $\mathrm{Fe}_{2} \mathrm{O}_{3}$ and $\mathrm{TiO}_{2}$ in different cultural eras and kilns 
Table 1: Chemical composition of the bodies from Longquan and Jingdezhen kilns

\begin{tabular}{|c|c|c|c|c|c|c|c|c|}
\hline Group & $\mathrm{Na}_{2} \mathrm{O}(\%)$ & $\mathrm{MgO}(\%)$ & $\mathrm{Al}_{2} \mathrm{O}_{3}(\%)$ & $\mathrm{SiO}_{2}(\%)$ & $\mathrm{K}_{2} \mathrm{O}(\%)$ & $\mathrm{CaO}(\%)$ & $\mathrm{TiO}_{2}(\%)$ & $\mathrm{Fe}_{2} \mathrm{O}_{3}(\%)$ \\
\hline FLQ-M & $0.52(0.21)$ & $0.39(0.15)$ & $19.62(1.52)$ & $72.40(2.10)$ & $4.20(0.53)$ & $0.51(0.28)$ & $0.08(0.05)$ & $1.28(0.47)$ \\
\hline LQ-BS & $0.30(0.22)$ & $0.33(0.11)$ & $21.71(2.15)$ & $68.99(2.82)$ & $5.51(0.51)$ & $0.16(0.02)$ & $0.14(0.03)$ & $1.86(0.11)$ \\
\hline LQ-NS & $0.35(0.19)$ & $0.36(0.12)$ & $22.62(2.30)$ & $67.85(2.72)$ & $5.16(0.76)$ & $0.23(0.12)$ & $0.15(0.08)$ & $2.28(0.57)$ \\
\hline LQ-Y & $0.37(0.21)$ & $0.36(0.11)$ & $23.55(1.74)$ & $67.06(2.26)$ & $5.13(1.07)$ & $0.16(0.03)$ & $0.15(0.08)$ & $2.23(0.50)$ \\
\hline LQ-M & $0.30(0.12)$ & $0.19(0.02)$ & $23.23(1.13)$ & $67.44(1.83)$ & $5.54(0.48)$ & $0.15(0.03)$ & $0.09(0.02)$ & $2.06(0.30)$ \\
\hline
\end{tabular}

Table 2: Chemical composition of the glaze from Longquan and Jingdezhen kilns

\begin{tabular}{lllllllll}
\hline Group & $\mathrm{Na}_{2} \mathrm{O}(\%)$ & $\mathrm{MgO}(\%)$ & $\mathrm{Al}_{2} \mathrm{O}_{3}(\%)$ & $\mathrm{SiO}_{2}(\%)$ & $\mathrm{K}_{2} \mathrm{O}(\%)$ & $\mathrm{CaO}(\%)$ & $\mathrm{TiO}_{2}(\%)$ & $\mathrm{Fe}_{2} \mathrm{O}_{3}(\%)$ \\
\hline FLQ-M & $1.01(0.46)$ & $0.41(0.25)$ & $12.87(0.67)$ & $70.21(2.16)$ & $5.08(0.95)$ & $7.76(2.31)$ & $0.09(0.04)$ & $1.57(0.49)$ \\
LQ-BS & $0.34(0.14)$ & $0.65(0.25)$ & $13.42(1.19)$ & $68.64(1.81)$ & $4.13(0.57)$ & $10.75(1.89)$ & $0.07(0.01)$ & $0.99(0.24)$ \\
LQ-NS & $0.34(0.27)$ & $0.58(0.22)$ & $13.56(0.65)$ & $70.43(1.84)$ & $4.89(0.91)$ & $8.15(2.49)$ & $0.08(0.03)$ & $0.96(0.26)$ \\
LQ-Y & $0.43(0.19)$ & $0.47(0.05)$ & $12.84(1.13)$ & $71.81(2.43)$ & $5.38(1.12)$ & $6.86(3.22)$ & $0.07(0.04)$ & $1.14(0.43)$ \\
LQ-M & $0.31(0.12)$ & $0.55(0.27)$ & $12.90(6.78)$ & $72.13(40.15)$ & $5.67(2.54)$ & $6.20(1.94)$ & $0.07(0.02)$ & $1.17(0.42)$ \\
\hline
\end{tabular}


Table 3: Sintering temperature of typical samples of celadon from Jingdezhen and Longquan kilns

\begin{tabular}{llc}
\hline Number & Kiln Location & Sintering Temperature $\left({ }^{\circ} \mathrm{C}\right)$ \\
\hline LQ-BS-3 & Longquan (Dayao) & $1182 \pm 20$ \\
LQ-NS-1 & Longquan & $1180 \pm 20$ \\
LQ-NS-1 & Longquan (Dayao) & $1206 \pm 20$ \\
LQD-01 & Longquan (Dayao) & $1181 \pm 20$ \\
LQD-04 & Longquan (Dayao) & $1171 \pm 20$ \\
LQ-Y-1 & Longquan (Dayao) & $1073 \pm 20$ \\
LQD-07 & Longquan (Dayao) & $1187 \pm 20$ \\
LQ-M-2 & Longquan (Dayao) & $1157 \pm 20$ \\
ML-1 & Longquan & $1230 \pm 20$ \\
FLQ-3 & Jingdezhen & $1159 \pm 20$ \\
FLQ-5 & Jingdezhen & $1167 \pm 20$ \\
FLQ-8 & Jingdezhen & $1132 \pm 20$ \\
FLQ-12 & Jingdezhen & $1101 \pm 20$ \\
\hline
\end{tabular}


Table 4: Variable importance for elements in body

\begin{tabular}{ccc}
\hline Element & MDA & MDG \\
\hline $\mathrm{ZrO}_{2}$ & 0.0704 & 4.0653 \\
$\mathrm{Fe}_{2} \mathrm{O}_{3}$ & 0.0515 & 3.7851 \\
$\mathrm{CaO}$ & 0.0398 & 2.7215 \\
$\mathrm{Y}_{2} \mathrm{O}_{3}$ & 0.0386 & 2.3640 \\
$\mathrm{MgO}$ & 0.0012 & 1.9451 \\
$\mathrm{Rb}_{2} \mathrm{O}$ & 0.0193 & 1.9348 \\
$\mathrm{SiO}_{2}$ & 0.0067 & 1.7819 \\
$\mathrm{~K}_{2} \mathrm{O}$ & 0.0080 & 1.5509 \\
$\mathrm{Al}_{2} \mathrm{O}_{3}$ & 0.0050 & 1.5311 \\
$\mathrm{P}_{2} \mathrm{O}_{5}$ & 0.0022 & 1.4201 \\
$\mathrm{Na}_{2} \mathrm{O}$ & -0.0016 & 1.2934 \\
$\mathrm{MnO}$ & -0.0008 & 1.2618 \\
$\mathrm{TiO}_{2}$ & -0.0004 & 1.2286 \\
$\mathrm{PbO}_{2}$ & -0.0021 & 1.1083 \\
$\mathrm{ZnO}$ & 0.0013 & 0.8112 \\
$\mathrm{CuO}$ & -0.0042 & 0.7016 \\
$\mathrm{SrO}$ & -0.0015 & 0.4448 \\
\hline
\end{tabular}


Table 5: Variable importance for elements in glaze

\begin{tabular}{ccc}
\hline Element & MDA & MDG \\
\hline $\mathrm{SrO}$ & 0.0781 & 4.3968 \\
$\mathrm{ZrO}_{2}$ & 0.0644 & 3.3247 \\
$\mathrm{Rb}_{2} \mathrm{O}$ & 0.0235 & 2.2258 \\
$\mathrm{Na}_{2} \mathrm{O}$ & 0.0133 & 2.1794 \\
$\mathrm{CaO}$ & 0.0047 & 1.9602 \\
$\mathrm{MnO}$ & 0.0110 & 1.9423 \\
$\mathrm{P}_{2} \mathrm{O}_{5}$ & 0.0084 & 1.7970 \\
$\mathrm{~K}_{2} \mathrm{O}$ & 0.0033 & 1.6330 \\
$\mathrm{Fe}_{2} \mathrm{O}_{3}$ & 0.0008 & 1.4497 \\
$\mathrm{SiO}_{2}$ & -0.0002 & 1.4034 \\
$\mathrm{Al}_{2} \mathrm{O}_{3}$ & -0.0023 & 1.3054 \\
$\mathrm{MgO} \mathrm{O}$ & -0.0017 & 1.2400 \\
$\mathrm{Y}_{2} \mathrm{O}_{3}$ & 0.0092 & 1.1379 \\
$\mathrm{ZnO}$ & 0.0008 & 1.1042 \\
$\mathrm{TiO}_{2}$ & 0.0036 & 1.0291 \\
$\mathrm{CuO}$ & -0.0014 & 0.9623 \\
$\mathrm{PbO}_{2}$ & -0.0012 & 0.7448 \\
\hline
\end{tabular}


Table 6: Chemical composition of refined mud from Longquan and Jingdezhen

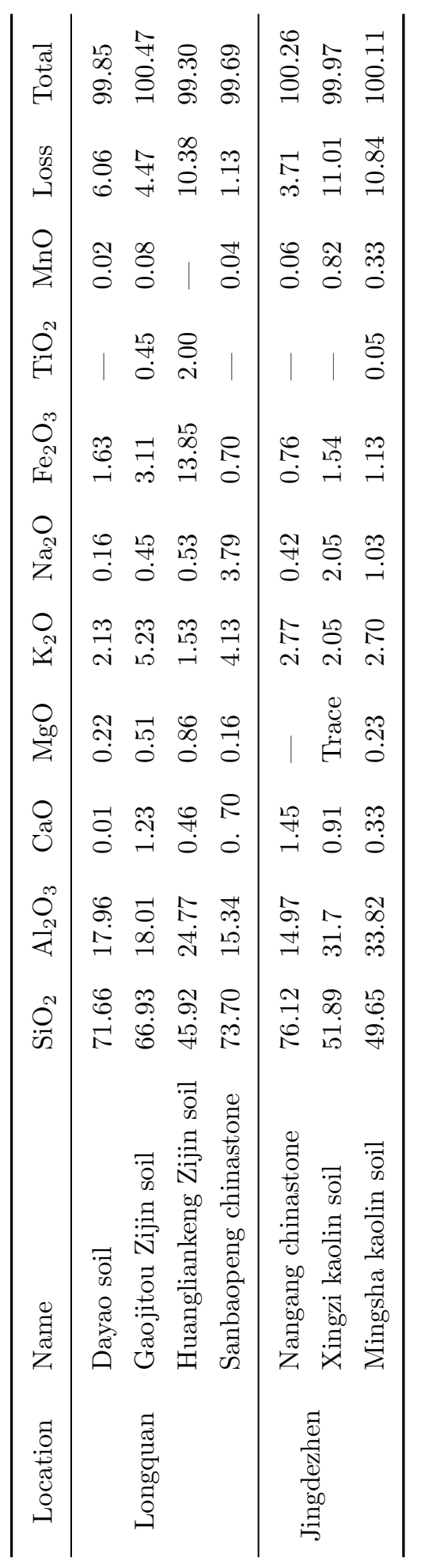

